\title{
OPTIMISING THE CARE OF OSCILLATED NEONATES BY OBTAINING ACCURATE INCUBATOR SCALE WEIGHTS
}

\section{U. El-Kafrawy}

NNU, Salford Royal Hospital, Manchester, UK

Background: Accurate weight measurement facilitates optimum care of the preterm infant, but can be problematic. Disconnection from the oscillator to weigh on standalone scales risks destabilising sick infants, whilst incubator scales are inaccurate when manikins are oscillated (see manikin study). We developed an adjustable device, to use in conjunction with incubator scales, to eliminate this problem.

Aim: To compare the accuracy, when weighing oscillated infants, of incubator scales alone to incubator scales in conjunction with the device.

Methodology: Infants were weighed while connected to the oscillator using the incubator scales alone then in combination with the device. A gold standard 'true weight' was obtained for comparison by disconnection from the ventilator and weighing on standalone scales.

45 sets of weights were recorded on 29 oscillated infants.

Results: The data were analysed using Stats direct, and displayed using the Bland Altman method. With incubator scales alone, the mean error in weight was $58.6 \mathrm{~g}$ (limits of agreement -30 to $+138 \mathrm{~g}$ ). With the adjustable device mean error $-4.6 \mathrm{~g}$ (limits of agreement -51 to $+42 \mathrm{~g}$ ). An F-test for variance showed that the improvement in accuracy was statistically significant $(\mathrm{p}<0.0001)$.

10 of the infants had a series of weights obtained. No number could be factored in to get a meaningful trend weight.

Conclusions: Incubator scales are not accurate for trend or individual weights in oscillated babies. An attachable device to ventilator tubing can be used to correct the error in weights obtained from incubator scales in oscillated neonates and obviate the risks of disconnection. 